\title{
Flavor from the double tetrahedral group without supersymmetry: Flavorful axions and neutrinos
}

\author{
Christopher D. Carone* and Marco Merchand ${ }^{\dagger}$ \\ High Energy Theory Group, Department of Physics, College of William and Mary, \\ Williamsburg, Virginia 23187-8795, USA
}

(Received 29 April 2019; published 8 August 2019)

\begin{abstract}
We extend the work of Carone, Chaurasia and Vasquez on nonsupersymmetric models of flavor based on the double tetrahedral group. Three issues are addressed: (1) the sector of flavor-symmetry-breaking fields is simplified and their potential studied explicitly, (2) a flavorful axion is introduced to solve the strong $C P$ problem and (3) the model is extended to include the neutrino sector. We show how the model can accommodate the strong hierarchies manifest in the charged fermion Yukawa matrices, while predicting a qualitatively different form for the light neutrino mass matrix that is consistent with observed neutrino mass squared differences and mixing angles.
\end{abstract}

DOI: $10.1103 /$ PhysRevD.100.035006

\section{INTRODUCTION}

The structure of the fermion Yukawa couplings in the standard model may result from the sequential breaking of a horizontal discrete family symmetry. Long ago, Aranda, Carone and Lebed [1,2] showed how the double tetrahedral group $T^{\prime}$ could be used to construct successful supersymmetric flavor models that are similar to those based on $\mathrm{U}(2)$ symmetry $[3,4]$, with or without the assumption of conventional supersymmetric grand unification. For other early work on $T^{\prime}$ as a flavor symmetry, see Ref. [5]. Many other authors have since explored the use of $T^{\prime}$ symmetry in models that aim to address the flavor structure of the standard model [6].

Much of the work on $T^{\prime}$ flavor models has assumed weakscale supersymmetry, to stabilize the hierarchy between the weak scale and the grand unified or Planck scale. Over the past decade, however, there has been no direct evidence for superpartners at the LHC, nor indirect evidence in the form of a convincing pattern of deviations from the predictions of the standard model for some subset of its observables. While one cannot exclude the possibility that supersymmetry is present and just beyond the reach of current experiments (a statement that applies to any new physics that has a decoupling limit), the current state of affairs has motivated a greater open-mindedness toward consideration of

\footnotetext{
*dcaro@wm.edu

†mamerchandmedi@email.wm.edu
}

Published by the American Physical Society under the terms of the Creative Commons Attribution 4.0 International license. Further distribution of this work must maintain attribution to the author(s) and the published article's title, journal citation, and DOI. Funded by SCOAP ${ }^{3}$. nonsupersymmetric extensions of the standard model. For example, the possibility that the standard model could arise consistently from a string theory without supersymmetry has been discussed in Ref. [7]. The hierarchies between mass scales might result from dynamical mechanisms (for example, cosmic relaxation [8] or Nnaturalness [9]), or anthropic selection [10]. On the other hand, the fundamental mass scales found in nature may simply be random and fine tuned, for reasons that are obscure to us at present. In this work, we assume the absence of supersymmetry and focus on phenomenological issues, while remaining agnostic on the question of naturalness.

The purpose of the present work is to further explore the possibility of nonsupersymmetric models of flavor based on $T^{\prime}$ symmetry, following a study by Carone, Chaurasia and Vasquez [11]. In Ref. [11], a nonsupersymmetric $T^{\prime}$ model was presented in which the flavor scale $M_{F}$ was treated as a free parameter. (There is less motivation to link the flavor scale to a grand unified scale in a framework where the gauge couplings do not automatically unify.) Global fits were performed to the fermion masses and Cabibbo-Kobayashi-Maskawa (CKM) mixing angles, taking into account the nonsupersymmetric running of the Yukawa matrices between the scale $M_{F}$ and the weak scale. It was found that the model was viable for a wide range of $M_{F}$; this scale could be as high as the Planck scale or as low as the minimum allowed by the flavor-changing-neutralcurrent constraints on the heavy, flavor-sector particles with masses of order $M_{F}$. At the lower end of this range, flavorsector fields, such as the physical components of the flavon fields that spontaneously break the $T^{\prime}$ symmetry, can potentially have observable consequences.

Here we go beyond the work of Ref. [11] in a number of ways: (i) we present a simplification of the model involving 
a smaller number of flavor-symmetry-breaking fields. While simplicity may be desirable by itself, the smaller field content allows a less cumbersome study of the flavon potential that leads to the spontaneous breaking of the flavor symmetry, so that we can confirm the assumed pattern of symmetry breaking and study the spectrum of scalar states. (ii) We address the strong $C P$ problem by promoting an Abelian factor that is required in the model from a $Z_{3}$ symmetry to an anomalous $\mathrm{U}(1)$ symmetry. This leads to a flavorful axion [12] (also called a flaxion [13], or axiflavon [14-16], in the recent literature), which leads to more stringent lower bounds on the flavor scale $M_{F}$ than in our previous study, as well as new avenues for discovery. (The idea of flavored axions appeared first in Ref. [17] and was explored subsequently by a number of authors [18].) The possibility of flavored axions due to a continuous Abelian factor in a $T^{\prime}$ flavor model was considered in a supersymmetric model in Ref. [19]; the present work gives a simple, nonsupersymmetric realization of this possibility. (iii) We extend the model to include the neutrino sector. As we describe later, one model building difficulty that we must overcome is to explain how the small symmetrybreaking parameters that lead to pronounced hierarchies in the charged fermion Yukawa matrices lead to much less pronounced hierarchies in the neutrino mass matrix (as indicated, for example, by the two large mixing angles). Our model will show how this outcome can be achieved.

Our paper is organized as follows: in Sec. II we present the model and establish our notation. We study the flavon potential including the vacuum alignment and the spectrum of scalar states. We also present a global fit of the charged fermion masses and mixing angles, analogous to the one presented in Ref. [11]. We address the strong $C P$ problem in Sec. III and identify the flavored axion couplings to SM particles. Bounds on the axion decay constant from flavor changing decays are given. In Sec. IV we address the neutrino sector and introduce a type-I seesaw mechanism with three right-handed neutrinos. In Sec. V, we summarize our conclusions.

\section{THE MODEL}

We assume the flavor symmetry $G_{F}=T^{\prime} \times Z_{3} \times U(1)$, where the last factor is anomalous and will allow for the existence of a flavorful axion. We do not review the group theory of $T^{\prime}$, which was discussed in some detail in Ref. [2] (including a useful appendix on Clebsch-Gordan factors), and reviewed again in Ref. [11]. We refer the reader to those references for details. The flavor-symmetry-breaking sector consists of three complex scalar fields $A, s$, and $\phi$, in the $\mathbf{1}^{0-}, \mathbf{1}^{00}$, and $\mathbf{2}^{0+}$ representations of $T^{\prime} \times Z_{3}$, using the notation of Ref. [2]. Notably, the triplet flavon $S$ of Ref. [11] has been omitted; the model is nonetheless viable, as we will discuss below. The complete field content and charge assignments for the model are shown in Table I.

Since the standard model fermions are charged under $G_{F}$, the Yukawa couplings, aside from that of the top quark, arise via higher-dimension operators involving the flavon fields. These are suppressed by appropriate powers of the flavor scale $M_{F}$, the cut off of the low-energy effective theory. When the flavon fields acquire vacuum expectation values (vevs), these operators depend on the ratios

$\langle\phi\rangle / M_{F} \equiv\left[\begin{array}{l}\epsilon \\ 0\end{array}\right],\langle A\rangle / M_{F} \equiv \epsilon^{\prime}$, and $\langle s\rangle / M_{F} \equiv \rho$

After flavor-symmetry breaking, the following Yukawa textures are generated:

$$
\begin{gathered}
Y_{U} \sim\left(\begin{array}{ccc}
0 & u_{1} \epsilon^{\prime} & 0 \\
-u_{1} \epsilon^{\prime} & u_{2} \epsilon^{2} & u_{3} \epsilon \\
0 & u_{4} \epsilon & u_{5}
\end{array}\right), \\
Y_{D} \sim\left(\begin{array}{ccc}
0 & d_{1} \epsilon^{\prime} & 0 \\
-d_{1} \epsilon^{\prime} & d_{2} \epsilon^{2} & d_{3} \epsilon \rho \\
0 & d_{4} \epsilon & d_{5} \rho
\end{array}\right), \\
Y_{E} \sim\left(\begin{array}{ccc}
0 & l_{1} \epsilon^{\prime} & 0 \\
-l_{1} \epsilon^{\prime} & l_{2} \epsilon^{2} & l_{3} \epsilon \\
0 & l_{4} \epsilon \rho & l_{5} \rho
\end{array}\right) .
\end{gathered}
$$

Here the $u_{i}, d_{i}$ and $l_{i}$ are (in general complex) $\mathcal{O}(1)$ parameters and only the leading-order expressions are presented. The nonzero entries differ in two ways from the textures of Ref. [11]: the 2-2 entries above are $\mathcal{O}\left(\epsilon^{2}\right)$, rather than $\mathcal{O}(\epsilon)$, due to the absence of the $T^{\prime}$-triplet flavon. However, the factors of $\rho$ appear in different locations, so that the end results are qualitatively similar. For example, the suppression of the 1-2 block of $Y_{u}$ in Ref. [11] by an overall factor of $\rho$ is mimicked here by the higher-order 2-2 entry and the proportionally smaller numerical value of $\epsilon^{\prime}$,

TABLE I. Charge assignments. The index $a=1,2$ is a generation label. The first four columns correspond to complex scalar fields, while the remainder are either right-handed standard model fermion fields or Dirac adjoints of left-handed ones.

\begin{tabular}{lcccccccccccccc}
\hline \hline & $\mathrm{A}$ & $s$ & $\phi$ & $H$ & $\bar{Q}_{L}^{a}$ & $\bar{Q}_{L}^{3}$ & $d_{R}^{a}$ & $d_{R}^{3}$ & $u_{R}^{a}$ & $u_{R}^{3}$ & $\bar{L}^{a}$ & $\bar{L}^{3}$ & $e_{R}^{a}$ & $e_{R}^{3}$ \\
\hline$T^{\prime} \times Z_{3}$ & $\mathbf{1}^{\mathbf{0 -}}$ & $\mathbf{1}^{\mathbf{0 0}}$ & $\mathbf{2}^{\mathbf{0}+}$ & $\mathbf{1}^{\mathbf{0 0}}$ & $\mathbf{2}^{\mathbf{0}-}$ & $\mathbf{1}^{\mathbf{0 0}}$ & $\mathbf{2}^{\mathbf{0}-}$ & $\mathbf{1}^{\mathbf{0 0}}$ & $\mathbf{2}^{\mathbf{0}-}$ & $\mathbf{1}^{\mathbf{0 0}}$ & $\mathbf{2}^{\mathbf{0}-}$ & $\mathbf{1}^{\mathbf{0 0}}$ & $\mathbf{2}^{\mathbf{0}-}$ & $\mathbf{1}^{\mathbf{0 0}}$ \\
$U(1)$ & 0 & 1 & 0 & 0 & 0 & 0 & 0 & -1 & 0 & 0 & 0 & -1 & 0 & 0 \\
\hline \hline
\end{tabular}


as we will see later. We also note that there will be $C P$ violation in the model even if all the operator coefficients defined at the level of the Lagrangian are real, due to imaginary numbers in Clebsch-Gordan coefficients; these would lead, for example, to factors of $i$ in the 2-2 entries of $Y_{U}, Y_{D}$ and $Y_{E}$. In general, however, all operator coefficients are themselves complex, and the 10 phase degrees of freedom in $Y_{U}$ and $Y_{D}$ can be used to obtain the desired CKM phase rather easily. In light of this, and to simplify our subsequent numerical analysis, we have chosen all the operator phases so that the parameters shown in Eqs. (2.2)-(2.4) are real, and omit the CKM phase from our global fit in Sec. II B.

\section{A. The flavon potential}

In this subsection, we consider the flavon potential, to confirm that the pattern of vevs assumed in Eq. (2.1) can be achieved and to study the spectrum of physical scalar states. We will do this by assuming the desired vev pattern, and imposing the extremization conditions on the potential to fix some of its otherwise free parameters. We then check the second-derivative matrix of the potential for positive definiteness. To simplify the discussion, we exclude the $s$ field, since it is a trivial singlet under the non-Abelian discrete flavor group and it is straightforward to write down a potential involving $s$ alone that provides for its vev. Including terms that couple $s$ to the other fields, e.g., $|s|^{2}\left|\phi^{2}\right|$, will not qualitatively change our results providing that their couplings are not too large, which is good enough for a proof of principle. We are particularly interested in accidental global symmetries that arise in the potential as a consequence of the $T^{\prime} \times Z_{3}$ discrete symmetry. These lead to pseudo-Goldstone bosons whose masses arise via higher-dimension operators. We estimate the masses of these states to confirm that they are not so light that their phenomenological consequences need to be taken into account. In this case, the only light state that will have interesting flavor-changing physics will be a single flavorful axion associated with the $s$ field.

The most general scalar potential for a singlet and a doublet transforming as $A \sim \mathbf{1}^{\mathbf{0}-}, \phi \sim \mathbf{2}^{\mathbf{0}+}$ under $T^{\prime} \times Z_{3}$, respectively, is given by

$$
V=V_{A}+V_{\phi}+V_{A \phi}
$$

where

$$
\begin{gathered}
V_{A}=m_{A}^{2}|A|^{2}+\mu\left(A^{3}+A^{* 3}\right)+\lambda_{A}|A|^{4}, \\
V_{\phi}=m_{\phi}^{2}|\phi|^{2}+\lambda_{\phi}|\phi|^{4},
\end{gathered}
$$

$$
V_{A \phi}=\lambda_{A \phi}|A|^{2}|\phi|^{2} .
$$

Note that this potential has an accidental $U(2)_{\phi}$ global symmetry as well as an additional $U(1)_{A}$ symmetry in the limit $\mu \rightarrow 0$. We parametrize the fields in terms of their real degrees of freedom

$$
A=\frac{1}{\sqrt{2}}\left(A_{1}+i A_{2}\right)
$$

and

$$
\phi=\frac{1}{\sqrt{2}}\left(\begin{array}{l}
\phi_{11}+i \phi_{12} \\
\phi_{21}+i \phi_{22}
\end{array}\right) .
$$

The Yukawa textures in Eqs. (2.2)-(2.4) are reproduced provided only the following real fields develop vevs:

$$
\left\langle\phi_{11}\right\rangle / \sqrt{2}=\epsilon M_{F} \quad \text { and } \quad\left\langle A_{1}\right\rangle / \sqrt{2}=\epsilon^{\prime} M_{F} .
$$

The location of a local minimum of the potential is determined by six first-derivative equations, corresponding to the six real scalar fields in Eqs. (2.9) and (2.10). However, for the assumed vev pattern, only two of these equations are nonvanishing,

$$
\begin{aligned}
\left.\frac{\partial V}{\partial A_{1}}\right|_{\mathrm{vev}} & =\sqrt{2} \epsilon^{\prime} M_{F}\left(m_{A}^{2}+2 \epsilon^{\prime 2} M_{F}^{2} \lambda_{A}+\epsilon^{2} M_{F}^{2} \lambda_{A \phi}+3 \epsilon^{\prime} M_{F} \mu\right) \\
& =0 \\
\left.\frac{\partial V}{\partial \phi_{11}}\right|_{\mathrm{vev}} & =\sqrt{2} \epsilon M_{F}\left(m_{\phi}^{2}+\epsilon^{\prime 2} M_{F}^{2} \lambda_{A \phi}+2 \epsilon^{2} M_{F}^{2} \lambda_{\phi}\right)=0
\end{aligned}
$$

where the subscript "vev" indicates that the fields have been set to their vevs, those shown in Eq (2.11) with all others vanishing. For a given choice of the dimensionless couplings, Eqs. (2.12) and (2.13) allow us to determine the mass parameters

$$
\begin{gathered}
m_{A}^{2}=-2 \epsilon^{\prime 2} M_{F}^{2} \lambda_{A}-\epsilon^{2} M_{F}^{2} \lambda_{A \phi}-3 \epsilon^{\prime} M_{F} \mu, \\
m_{\phi}^{2}=-\epsilon^{\prime 2} M_{F}^{2} \lambda_{A \phi}-2 \epsilon^{2} M_{F}^{2} \lambda_{\phi} .
\end{gathered}
$$

To obtain the mass spectrum, we construct the second derivative matrix for the potential in terms of the six real scalar fields, evaluated with the assumed vevs, and with mass parameters fixed by Eqs. (2.14) and (2.15). In the basis $\left(\phi_{11}, A_{1}, A_{2}, \phi_{12}, \phi_{21}, \phi_{22}\right)$ we find 


$$
m_{\text {scalar }}^{2}=\left(\begin{array}{cccccc}
4 \epsilon^{2} M_{F}^{2} \lambda_{\phi} & 2 \epsilon \epsilon^{\prime} M_{F}^{2} \lambda_{A \phi} & 0 & 0 & 0 & 0 \\
2 \epsilon \epsilon^{\prime} M_{F}^{2} \lambda_{A \phi} & \epsilon^{\prime} M_{F}\left(4 \epsilon^{\prime} M_{F} \lambda_{A}+3 \mu\right) & 0 & 0 & 0 & 0 \\
0 & 0 & -9 \epsilon^{\prime} M_{F} \mu & 0 & 0 & 0 \\
0 & 0 & 0 & 0 & 0 & 0 \\
0 & 0 & 0 & 0 & 0 & 0 \\
0 & 0 & 0 & 0 & 0 & 0
\end{array}\right)
$$

The three nonvanishing eigenvalues of the mass squared matrix are positive, provided that $\mu<0$ and (assuming $\epsilon, \epsilon^{\prime}$ and $\lambda_{\phi}$ are positive)

$|\mu|<\frac{\epsilon^{\prime} M_{F}}{3 \lambda_{\phi}}\left(4 \lambda_{A} \lambda_{\phi}-\lambda_{A \phi}^{2}\right)$ and $|\mu|<\frac{4}{3} \frac{M_{F}}{\epsilon^{\prime}}\left(\epsilon^{2} \lambda_{\phi}+\epsilon^{\prime 2} \lambda_{A}\right)$,

which is easily arranged. The three massless states are expected from Goldstone's theorem, since the $\mathrm{U}(2)_{\phi}$ symmetry is spontaneously broken to a residual $\mathrm{U}(1)$ symmetry that rotates the second component of the $\phi$ column vector by a phase. However, these zero eigenvalues are lifted when one takes into account corrections to the potential from higher-dimension operators that break the accidental $\mathrm{U}(2)_{\phi}$ global symmetry. We find that the lowestorder operators that have this effect occur at dimension 6,

$$
\begin{aligned}
V_{h d}= & \frac{1}{M_{F}^{2}}\left(c_{61}\left[(\phi \phi)_{\mathbf{3}}(\phi \phi)_{\mathbf{3}}\right]_{\mathbf{3}}(\phi \phi)_{\mathbf{3}}+\text { H.c. }\right) \\
& +c_{62} \frac{1}{M_{F}^{2}}\left[\left(\phi^{*} \phi^{*}\right)_{\mathbf{3}}(\phi \phi)_{\mathbf{3}}\right]_{\mathbf{3}}\left(\phi^{*} \phi\right)_{\mathbf{3}}+\cdots,
\end{aligned}
$$

where the subscript indicates the $T^{\prime}$ representation of the given product, with Clebsch-Gordan factors left implicit. We have studied the eigenvalues of Eq. (2.16) numerically after including the additional potential terms in $V_{h d}$, and find that all the eigenvalues are positive and nonvanishing; the masses of the three pseudo-Goldstone bosons are of order $\epsilon^{2} M_{F}$. We will see later that the numerical values of our symmetry breaking parameters and our extension to the neutrino sector will imply that this scale corresponds to roughly $10^{12} \mathrm{GeV}$. We therefore do not expect meaningful phenomenological bounds on the three pseudo-Goldstone states. We note that there are also dimension- 5 operators that one can write down which correct the potential (e.g., $A^{3}|\phi|^{2}$ and $A^{3}|A|^{2}$ ) but these do not break the accidental $\mathrm{U}(2)_{\phi}$ symmetry and provide higher-order corrections to the eigenvalues that are already nonvanishing at lowest order.

\section{B. Fit to quarks and charged leptons}

In this subsection, we verify that the Yukawa textures in Eqs. (2.2)-(2.4) reproduce the correct masses and mixing angles for the charged fermions, by performing a global fit that takes into account running from a high scale (which we will take to be $4 \times 10^{16} \mathrm{GeV}$, to be consistent with our later discussion of the neutrino sector) down to the weak scale. This is the same analysis that was performed in Ref. [11] for an arbitrary $M_{F}$ scale, but is now modified to take into account the textures predicted in the present model. We take the model parameters $\left\{u_{i}, d_{i}, l_{i}, \epsilon, \epsilon^{\prime}, \rho\right\}$ to be real as a simplifying assumption since, as discussed earlier, there is no difficulty in accommodating a CKM phase if one allows an arbitrary phase parameter for every operator coefficient. The experimental inputs are the quark and lepton masses and CKM angles, which we associate with the scale $m_{Z}$ (i.e., we ignore weak scale threshold corrections). We seek solutions in which the order one parameters are in fact not far from one, while predictions for the observables, renormalized at the weak scale, are within two standard deviations of experimental values. Employing the same technique as Refs. [2,11], we construct a function $\tilde{\chi}$ whose minimization achieves this goal:

$$
\begin{aligned}
\tilde{\chi}^{2}= & \sum_{i=1}^{9}\left(\frac{m_{i}^{t h}-m_{i}^{\exp }}{\Delta m_{i}^{\exp }}\right)^{2}+\left(\frac{\left|V_{u s}^{t h}\right|-\left|V_{u s}^{\exp }\right|}{\Delta V_{u s}^{\exp }}\right)^{2} \\
& +\left(\frac{\left|V_{u b}^{t h}\right|-\left|V_{u b}^{\exp }\right|}{\Delta V_{u b}^{\exp }}\right)^{2}+\left(\frac{\left|V_{c b}^{t h}\right|-\left|V_{c b}^{\exp }\right|}{\Delta V_{c b}^{\exp }}\right)^{2} \\
& +\sum_{i=1}^{5}\left(\frac{\ln \left|u_{i}\right|}{\ln 3}\right)^{2}+\sum_{i=1}^{5}\left(\frac{\ln \left|d_{i}\right|}{\ln 3}\right)^{2}+\sum_{i=1}^{5}\left(\frac{\ln \left|\ell_{i}\right|}{\ln 3}\right)^{2} .
\end{aligned}
$$

The first four terms would be present in a conventional chisquared function, and place weight on how close the theoretical predictions for observables are to experimental observations, relative to the experimental error. The experimental errors are handled as in Refs. [2,11]: they are inflated to $1 \%$ of the central measured value if the error is smaller that this amount. This takes into account theoretical uncertainties (for example, two-loop running effects) that have been omitted. The remaining three terms of Eq. (2.19) 
TABLE II. Fit to the charged fermion masses and mixing angles. All masses are given in GeV. (Note that $m_{t}$ is the $\overline{\mathrm{MS}}$ mass, not the pole mass.) The value of the quantity $\tilde{\chi}^{2}$ defined in the text is 12.3 . Running from the flavor scale $M_{F}$ down to the $Z$ mass is taken into account, with $M_{F}=4 \times 10^{16} \mathrm{GeV}$, (see Sec. IV) chosen for the purpose of illustration.

\begin{tabular}{lcc}
\hline \multicolumn{3}{c}{ Best fit parameters } \\
\hline \multicolumn{2}{c}{$\epsilon=2.42 \times 10^{-2}, \epsilon^{\prime}=9.75 \times 10^{-5}, \rho=-1.38 \times 10^{-2}$} \\
\hline$u_{1}=1.22$ & $d_{1}=0.662$ & $\ell_{1}=0.612$ \\
$u_{2}=-0.671$ & $d_{2}=1.29$ & $\ell_{2}=0.643$ \\
$u_{3}=-2.26$ & $d_{3}=-1.02$ & $\ell_{3}=0.352$ \\
$u_{4}=-0.702$ & $d_{4}=-0.276$ & $\ell_{4}=2.40$ \\
$u_{5}=0.384$ & $d_{5}=0.376$ & $\ell_{5}=0.295$ \\
Observable & Expt. value from $[21]$ & Fit value \\
$m_{u}$ & $(2.2 \pm 0.45) \times 10^{-3}$ & $2.30 \times 10^{-3}$ \\
$m_{c}$ & $1.275 \pm 0.03$ & 1.274 \\
$m_{t}$ & $160 \pm 4.5$ & 160.0 \\
$m_{d}$ & $(4.7 \pm 0.4) \times 10^{-3}$ & $5.42 \times 10^{-3}$ \\
$m_{s}$ & $(9.5 \pm 0.6) \times 10^{-2}$ & $9.16 \times 10^{-2}$ \\
$m_{b}$ & $4.18 \pm 0.035$ & 4.17 \\
$m_{e}$ & $(5.11 \pm 1 \%) \times 10^{-4}$ & $5.11 \times 10^{-4}$ \\
$m_{\mu}$ & $0.106 \pm 1 \%$ & 0.106 \\
$m_{\tau}$ & $1.78 \pm 1 \%$ & 1.78 \\
$\left|V_{u s}\right|$ & $0.225 \pm 1 \%$ & 0.223 \\
$\left|V_{u b}\right|$ & $(3.65 \pm 0.12) \times 10^{-3}$ & $3.62 \times 10^{-3}$ \\
$\left|V_{c b}\right|$ & $(4.21 \pm 0.08) \times 10^{-2}$ & $4.17 \times 10^{-2}$ \\
\hline \hline & & \\
\hline & &
\end{tabular}

place weight on the coefficients having values that are order one, the expectation of naive dimensional analysis. Including these terms is equivalent to assuming that the coefficients are distributed with a log-normal distribution with mean 1 and standard deviation $\sigma=\ln (3) / 2$ such that the absolute value of an element drawn from the distribution has a $95 \%$ probability to lie in the range $[0.3,3]$. There are a total of 12 observables (nine masses and three mixing angles) and, given the stated constraints on the model parameters, the only three genuine free parameters, $\left\{\epsilon, \epsilon^{\prime}, \rho\right\}{ }^{1}$ Thus, we expect a good fit if $\tilde{\chi}^{2} \approx 9$. The best fit values together with the experimental and theoretical predictions are presented in Table II. We note that our successful results might be anticipated from the qualitatively similar Yukawa textures obtained in $U(2)$ flavor models [15,20], a further example of the similarities

\footnotetext{
${ }^{1}$ It should be stressed that the number of operator coefficients can exceed the number of observables without sacrificing predictivity because we work in an effective field theory framework where these coefficients are not free parameters, but are constrained by the requirement that they remain consistent with naive dimensional analysis. As in the seminal work of Ref. [2], as well as Ref. [11], this is imposed via the terms in the $\tilde{\chi}^{2}$ function that incorporate a preference for coefficients with magnitudes between $1 / 3$ and 3 . This choice eliminates the consideration of unnatural effective theories, including those that are not consistent with a perturbative operator expansion.
}

between $T^{\prime}$ models and $\mathrm{U}(2)$ models that was the focus of Refs. [1,2].

Finally, we note that global symmetries are expected to be broken by quantum gravity effects [22], but we can assume that there is an ultraviolet completion which allows the U(1) symmetry to arise as a consequence of the continuous and discrete gauge symmetries that are present in a more complete theory. Another concern in the present framework is that the breaking of discrete symmetries can lead to potential domain-wall problems. However, these can be rendered harmless it the domain regions are widely separated due to inflation. We will find later in Sec. III that $M_{F}$ is constrained to be sufficiently high so that any problems with domain walls may be eliminated via this mechanism.

\section{THE FLAVORFUL AXION}

The model we have presented includes a flavon field $s$, charged under the $\mathrm{U}(1)$ factor of the flavor group, which assures, for example, the correct values of the bottom quark and tau lepton Yukawa couplings. This U(1) also serves as a Peccei-Quinn (PQ) symmetry and its spontaneous breaking leads to a flavorful axion. Only the third generation righthanded down quark and the third generation left-handed lepton doublet have nontrivial charges under the $\mathrm{U}(1)$ symmetry (see Table I), but rotation to the mass eigenstate basis will induce axion couplings to fields of the first two generations. The axion is identified via the nonlinear representation

$$
s=\frac{v_{s}+\sigma}{\sqrt{2}} e^{i a / v_{s}} .
$$

The radial component $\sigma$ is a heavy degree of freedom and is integrated out of the low-energy effective field theory. The phase field $a$ is the Goldstone boson of the spontaneously broken $\mathrm{U}(1) \equiv \mathrm{U}(1)_{P Q}$ symmetry and is identified with the QCD axion. Nonperturbative QCD effects generate a potential for the axion, with the minimum corresponding to vanishing of the $\bar{\theta}$ parameter of $\mathrm{QCD}$, solving the strong $C P$ problem. For complete reviews on this subject see Refs. [23,24].

The axiflavon couplings to fermions originate from the following Yukawa couplings

$$
\mathcal{L}_{Y a}=-\left[\bar{Q}^{i} Y_{i 3}^{d} H d_{R}^{3}+\bar{L}^{3} Y_{3 j}^{e} H e_{R}^{j}\right] \frac{s}{M_{F}}+\text { H.c. },
$$

or more explicitly

$$
\begin{aligned}
\mathcal{L}_{Y a}= & -\left[d_{3} \bar{Q}^{a} \epsilon_{a b}\left(\frac{\left\langle\phi^{b}\right\rangle}{M_{F}}\right) H d_{R}^{3}+d_{5} \bar{Q}^{3} H d_{R}^{3}\right. \\
& \left.+l_{4} \bar{L}^{3} \epsilon_{a b}\left(\frac{\left\langle\phi^{b}\right\rangle}{M_{F}}\right) H e_{R}^{a}+l_{5} \bar{L}^{3} H e_{R}^{3}\right] \frac{v_{s} e^{i a / v_{s}}}{\sqrt{2} M_{F}}+\text { H.c., }
\end{aligned}
$$


where $\epsilon_{a b}, a, b=1,2$ is the Clebsch-Gordan matrix that allows one to combine two $\mathbf{2}^{0}$ representations of $T^{\prime}$ into a $\mathbf{1}^{0}$. Setting the $\phi$ flavon to its vev, one obtains

$$
\begin{aligned}
\mathcal{L}_{Y a}= & -\left[-d_{3} \epsilon \bar{Q}^{2} H d_{R}^{3}+d_{5} \bar{Q}^{3} H d_{R}^{3}-l_{4} \epsilon \bar{L}^{3} H e_{R}^{2}+l_{5} \bar{L}^{3} H e_{R}^{3}\right] \\
& \times \frac{v_{s} e^{i a / v_{s}}}{\sqrt{2} M_{F}}+\text { H.c. }
\end{aligned}
$$

Performing the usual nonlinear field redefinition of the third generation fermions

$$
d_{R}^{3} \rightarrow e^{-i a / v_{s}} d_{R}^{3}, \quad \bar{L}^{3} \rightarrow e^{-i a / v_{s}} \bar{L}^{3},
$$

we remove the axion entirely from the Yukawa sector, but instead induce derivative interactions coming from the original fermion kinetic terms. For the charged fermions, one finds

$$
\begin{aligned}
\mathcal{L} \supseteq & \frac{\partial_{\mu} a}{v_{s}}\left[\bar{d}_{i} \gamma^{\mu}\left(K_{d}^{\dagger}\right)_{i 3}\left(K_{d}\right)_{3 j} \frac{\left(1+\gamma_{5}\right)}{2} d_{j}\right. \\
& \left.+\bar{e}_{i} \gamma^{\mu}\left(U_{e}^{\dagger}\right)_{i 3}\left(U_{e}\right)_{3 j} \frac{\left(1-\gamma_{5}\right)}{2} e_{j}\right] .
\end{aligned}
$$

Here $K_{d}\left(U_{e}\right)$ is the right-handed (left-handed) rotation that diagonalize the Yukawa interactions, where in our conventions a generic Yukawa matrix $Y$ would be diagonalized by $Y=U_{L} Y^{\text {diag }} U_{R}^{\dagger}$. Notice that the axion interactions with the fermion mass eigenstates are in general not diagonal and therefore induce flavor-changing neutral currents (FCNC) at tree level. Flavon FCNCs are very well constrained experimentally $[25,26]$ and we will discuss these constraints in the next subsection. See Refs. [27-29] for other axion models with FCNCs at tree level.

While our phenomenological bounds will come from the couplings in Eq. (3.6), we give the axion couplings to two gauge fields here for completeness. After the anomalous chiral rotation in Eq. (3.5), the axion reappears in an effective interaction with the gluon field strength and its dual, namely

$$
\mathcal{L}=\frac{\alpha_{s}}{8 \pi} \frac{a}{v_{s}} N_{D W} G_{\mu \nu}^{a} \tilde{G}^{a \mu \nu}
$$

With the charge assignments of Table I, we obtain the domain-wall number

$$
N_{D W}=\left[2 \sum_{i} X_{Q}^{i}-\sum_{i} X_{u}^{i}-\sum_{i} X_{d}^{i}\right]=1
$$

where the $X_{a}$ represent the $\mathrm{U}(1)$ charges for left-handed and the right-handed fermion fields. Since $N_{D W}=1$, there is one minimum of the axion potential. We identify the axion decay constant as

$$
f_{a}=\left|v_{s} / N_{D W}\right| .
$$

The PQ charge assignments give rise to $\mathrm{U}(1)_{2}^{Y} \mathrm{U}(1)_{P Q}$ and $\mathrm{SU}(2)^{2} U(1)_{P Q}$ anomalies and therefore axion couplings to hypercharge and electroweak gauge bosons are induced, namely

$\mathcal{L} \supseteq \frac{g^{\prime 2}}{32 \pi^{2}} \frac{a}{v_{s}}\left(2 N_{B}\right) B_{\mu \nu} \tilde{B}^{\mu \nu}+\frac{g^{2}}{32 \pi^{2}} \frac{a}{v_{s}} N_{W} W_{\mu \nu}^{a} \tilde{W}^{a \mu \nu}$.

Rewriting this piece of the Lagrangian in the gauge boson mass eigenstate basis one obtains the axion couplings to photons

$$
\mathcal{L}_{\gamma a}=\frac{\alpha_{\mathrm{EM}}}{8 \pi} \frac{a}{v_{s}}\left(2 N_{B}+N_{W}\right) F_{\mu \nu} \tilde{F}^{\mu \nu}
$$

where in this model one obtains

$$
\begin{aligned}
N_{B}= & 3\left[2 \sum_{i}\left(\frac{1}{6}\right)^{2} X_{Q}^{i}-\sum_{i}\left(\frac{2}{3}\right)^{2} X_{u}^{i}-\sum_{i}\left(-\frac{1}{3}\right)^{2} X_{d}^{i}\right] \\
& +2 \sum_{i}\left(-\frac{1}{2}\right)^{2} X_{L}^{i}-\sum_{i}(-1)^{2} X_{e}^{i}=\frac{5}{6} \\
N_{W}= & \sum_{i} X_{L}^{i}+3 \sum_{i} X_{Q}^{i}=1
\end{aligned}
$$

and thus the ratio of the electromagnetic to color anomalies is

$$
\frac{2 N_{B}+N_{W}}{N_{D W}}=\frac{8}{3} .
$$

As noted in other flavored axion models that make the same prediction for this ratio [14], this is consistent with the predictions of the simplest Dine-Fischler-SrednickiZhitnitsky (DFSZ) axion models [23,24].

\section{A. Constraints from meson decays}

As can be seen from the axion couplings to fermions in Eq. (3.6), our model predicts flavor violating processes, e.g., heavy meson decays like $K^{+} \rightarrow \pi^{+} a$. The branching fraction for a generic meson two-body decay $P \rightarrow P^{\prime} a$ is given by [12]

$$
\begin{aligned}
\operatorname{BR}\left(P \rightarrow P^{\prime} a\right)= & \frac{1}{64 \pi \Gamma(P)} \frac{\left|\left(K_{d}\right)_{i 3}^{\dagger}\left(K_{d}\right)_{3 j}\right|^{2}}{f_{a}^{2}} \\
& \times m_{P}^{3}\left(1-\frac{m_{P^{\prime}}^{2}}{m_{P}^{2}}\right)^{3}\left|f_{+}(0)\right|^{2}
\end{aligned}
$$

where $P=\left(\bar{q}_{i} q\right), P^{\prime}=\left(\bar{q}_{j} q\right)$ and the indices $i j$ denote the constituent quarks. The function $f_{+}\left(q^{2}\right)$ is the form factor from hadronic physics calculations and $q=q_{P}-q_{P^{\prime}}$ is the 
momentum transfer to the axion; one may take $q^{2} \approx 0$ as the axion is very light. The axion mass is the same as a QCD axion, $m_{a} \approx 6 \times 10^{-6} \times\left(10^{12} \mathrm{GeV} / f_{a}\right) \mathrm{eV}$ [12]; we will see that the strongest bounds presented later in this section imply $m_{a} \lesssim 10^{-4} \mathrm{eV}$, while the neutrino model discussed in the next section corresponds to $m_{a} \approx 7 \times 10^{-9} \mathrm{eV}$.

Experimental bounds on different heavy mesons decays are summarized in Ref. [12]. In Table III, we quote the most relevant of these constraints and indicate the relevant experimental references. The precise numerical bounds that follow from the fit presented in Sec. II B are displayed in the last column of this table.

To understand our results qualitatively, it is useful to parametrize the rotation matrices that correspond to the fit in Table II in terms of powers of the Cabibbo angle $\lambda \approx 0.22$. We find numerically that $K_{d}$ and $U_{e}$ have the qualitative form

$K_{d} \sim\left(\begin{array}{ccc}1 & \lambda & \lambda^{5} \\ \lambda & 1 & 1 \\ \lambda & 1 & 1\end{array}\right) \quad$ and $U_{e} \sim\left(\begin{array}{ccc}1 & \lambda & \lambda^{5} \\ \lambda^{2} & 1 & 1 \\ \lambda^{2} & 1 & 1\end{array}\right)$

The relevant combinations that determine the results in Tables III and IV are

$$
\begin{aligned}
\left(K_{d}^{\dagger}\right)_{i 3}\left(K_{d}\right)_{3 j} & \sim\left(\begin{array}{ccc}
\lambda^{2} & \lambda & \lambda \\
\lambda & 1 & 1 \\
\lambda & 1 & 1
\end{array}\right) \text { and } \\
\left(U_{e}^{\dagger}\right)_{i 3}\left(U_{e}\right)_{3 j} & \sim\left(\begin{array}{ccc}
\lambda^{3} & \lambda^{2} & \lambda^{2} \\
\lambda^{2} & 1 & \lambda \\
\lambda^{2} & \lambda & 1
\end{array}\right) .
\end{aligned}
$$

The strongest bound in this model comes from the heavy meson decay $K^{+} \rightarrow \pi^{+} a$ giving

$$
f_{a}>6.3 \times 10^{10} \mathrm{GeV}
$$

Given the identification $f_{a}=\left|v_{s} / N_{D W}\right|=\sqrt{2}|\rho| M_{F}$, we can translate this to a bound on the flavor scale

$$
M_{F}>3.2 \times 10^{12} \mathrm{GeV}
$$

Axion mixing with neutral hadronic mesons does not lead to competitive bounds and will not be discussed here. See Ref. [12] for a treatment of these effects.

\section{B. Constraints from lepton decays}

From the axiflavon couplings in Eq. (3.6) one can also compute the branching fraction for leptonic decays, namely [12]

$$
\mathrm{BR}\left(e_{i} \rightarrow e_{j} a\right)=\frac{1}{32 \pi \Gamma\left(e_{i}\right)} \frac{m_{i}^{3}}{f_{a}^{2}}\left|\left(U_{e}^{\dagger}\right)_{i 3}\left(U_{e}\right)_{3 j}\right|^{2}\left(1-\frac{m_{j}^{2}}{m_{i}^{2}}\right)^{3} .
$$

The most stringent bound comes from the decay $\mu^{+} \rightarrow e^{+} a$ giving $f_{a}>1.7 \times 10^{8} \mathrm{GeV}$, which is not competitive with our earlier bound from charged kaon decays, Eq. (3.19).

One can also find bounds from lepton decays with a photon in the final state but it turns out that these are not stronger than the bounds we have already considered.

TABLE III. Experimental constraints on the branching fractions of heavy mesons decays (second column), derived bounds on the axion decay constant times flavor rotation matrix elements from Ref. [12] (third column) and lower bound on the axion decay constant using the numerical value of the matrix element from the fit presented in Sec. II B (fourth column).

\begin{tabular}{llll}
\hline \hline Decay & Branching ratio & Bound $\left(f_{a} / \mathrm{GeV}\right)$ & Bound from fit \\
\hline$K^{+} \rightarrow \pi^{+} a$ & $<0.73 \times 10^{-10}[30]$ & $>3.45 \times 10^{11}\left|\left(K_{d}^{\dagger}\right)_{23}\left(K_{d}\right)_{31}\right|$ & $f_{a}>6.3 \times 10^{10} \mathrm{GeV}$ \\
$K_{L}^{0} \rightarrow \pi^{0} a$ & $<5 \times 10^{-8}[31]$ & $>1.35 \times 10^{10}\left|\left(K_{d}^{\dagger}\right)_{23}\left(K_{d}\right)_{31}\right|$ & $f_{a}>2.5 \times 10^{9} \mathrm{GeV}$ \\
$B^{ \pm} \rightarrow \pi^{ \pm} a$ & $<4.9 \times 10^{-5}[32]$ & $>5.0 \times 10^{7}\left|\left(K_{d}^{\dagger}\right)_{33}\left(K_{d}\right)_{31}\right|$ & $f_{a}>7.4 \times 10^{6} \mathrm{GeV}$ \\
$B^{ \pm} \rightarrow K^{ \pm} a$ & $<4.9 \times 10^{-5}[32]$ & $>6.0 \times 10^{7}\left|\left(K_{d}^{\dagger}\right)_{33}\left(K_{d}\right)_{32}\right|$ & $f_{a}>2.8 \times 10^{7} \mathrm{GeV}$ \\
\hline \hline
\end{tabular}

TABLE IV. Experimental constraints on the branching fractions of lepton decays (second column), derived bounds on the axion decay constant times flavor rotation matrix elements from Ref. [12] (third column) and lower bound on the axion decay constant using the predicted numerical values from out fit (fourth column).

\begin{tabular}{lccc}
\hline \hline Decay & Branching ratio & Bound $\left(f_{a} / \mathrm{GeV}\right)$ & Bound from fit \\
\hline$\mu^{+} \rightarrow e^{+} a$ & $<1.0 \times 10^{-5}[33]$ & $>2.0 \times 10^{9}\left|\left(U_{e}^{\dagger}\right)_{23}\left(U_{e}\right)_{31}\right|$ & $f_{a}>1.7 \times 10^{8} \mathrm{GeV}$ \\
$\tau^{+} \rightarrow e^{+} a$ & $<1.5 \times 10^{-2}[34]$ & $>1.3 \times 10^{6}\left|\left(U_{e}^{\dagger}\right)_{33}\left(U_{e}\right)_{31}\right|$ & $f_{a}>5.3 \times 10^{4} \mathrm{GeV}$ \\
$\tau^{+} \rightarrow \mu^{+} a$ & $<2.6 \times 10^{-2}[34]$ & $>9.9 \times 10^{5}\left|\left(U_{e}^{\dagger}\right)_{33}\left(U_{e}\right)_{32}\right|$ & $f_{a}>3.9 \times 10^{5} \mathrm{GeV}$ \\
\hline \hline
\end{tabular}




\section{NEUTRINO SECTOR}

In this section, we consider how our model may be extended to explain the observed neutrino masses and mixing angles. In doing so, we face an immediate challenge: how can we explain two large neutrino mixing angles in a theory where symmetry breaking is achieved through two small parameters, $\epsilon$ and $\epsilon^{\prime}$, that are of order $10^{-2}$ and $10^{-4}$, respectively? A similar problem presents itself when one considers the neutrino mass squared differences. The smallness of the overall neutrino mass scale can be explained via the seesaw mechanism; we will implement a type-I seesaw mechanism below, involving three right-handed neutrinos. Choice of the right-handed neutrino mass scale allows us to fix one of the observed neutrino mass squared differences, for example, $\Delta m_{32}^{2}$; what is then determined by the symmetry breaking parameters is the ratio $\Delta m_{32}^{2} / \Delta m_{21}^{2}$, which is found experimentally to be $33.3 \pm 1.03$ [21], assuming a normal, rather than inverted, neutrino mass hierarchy (which is the case on our model). One would expect that the theoretical prediction for $\Delta m_{32}^{2} / \Delta m_{21}^{2}$ is proportional to ratios of powers of $\epsilon$ and $\epsilon^{\prime}$; if this quantity is not $\mathcal{O}(1)$, then one finds typically that the predicted value is either much too large or too small to account for the experimental value. This is a consequence of the small and distinctly hierarchical values of $\epsilon$ and $\epsilon^{\prime}$. One cannot decouple the problem of the neutrino sector from our results in the charged fermion sector because the left-handed neutrinos must have the same flavor charge assignments as their charged partners within each weak SU(2) doublet. As a consequence, there is a real possibility that the flavor structure of the theory might only be consistent with neutrino masses and mixing angles that are strongly hierarchical, which would make our previous findings in the charged fermion sector of dubious value. It is therefore important to demonstrate explicitly that an extension to the neutrino sector (via appropriate charge assignments for right-handed neutrinos) is possible that avoids this problem. We present such a solution in this section.

We note that if the ratio $\Delta m_{32}^{2} / \Delta m_{21}^{2}$ is approximately independent of $\epsilon$ and $\epsilon^{\prime}$, then it is a function of the order one coefficients in the theory alone. In this case, a value of 33.3 can be obtained for a rather mundane reason: The seesaw formula tells us that the mass matrix of the light, lefthanded neutrino mass eigenstates is given by

$$
M_{L L} \approx M_{L R} M_{R R}^{-1} M_{L R}^{\dagger}
$$

which implies that the eigenvalues of $M_{L L}$ will typically be of cubic order in quantities of $\mathcal{O}(1)$, either operator coefficients or their inverse. Here, $M_{L R}$ represents the neutrino Dirac mass matrix, while $M_{R R}$ is the Majorana mass matrix for the righthanded neutrinos. The numerator and denominator of $\Delta m_{32}^{2} / \Delta m_{21}^{2}$ then each depend on terms that are of sixth order in quantities that are $\mathcal{O}(1)$, with each typically falling somewhere between $1 / 3$ and 3 in absolute value, given our earlier assumptions. Noting that $1.8^{6} \approx 34$, one can understand how easy it is to take input matrices with coefficients that are of $\mathcal{O}(1)$ and still obtain a mass-squared-difference ratio that is consistent with the experimental value. This observation is relevant to our solution below.

We introduce three right-handed neutrinos that are uncharged under the Peccei-Quinn symmetry and have $T^{\prime} \times Z_{3}$ charges

$$
\nu_{R}^{1} \sim \mathbf{1}^{0-}, \quad \text { and } \quad \nu_{R}^{2,3} \sim \mathbf{1}^{\mathbf{0 0}} .
$$

The Dirac and Majorana mass matrices have the following $T^{\prime} \times Z_{3} \times U(1)$ transformation properties

$$
\begin{aligned}
& M_{L R} \sim\left(\begin{array}{cc|c}
\mathbf{2}^{0-} & \mathbf{2}^{0+} & \mathbf{2}^{0+} \\
\hline \mathbf{1}_{+1}^{0+} & \mathbf{1}_{+1}^{00} & \mathbf{1}_{+1}^{00}
\end{array}\right) \text { and } \\
& M_{R R} \sim\left(\begin{array}{cc|c}
\mathbf{1}^{0-} & \mathbf{1}^{0+} & \mathbf{1}^{0+} \\
\mathbf{1}^{0+} & \mathbf{1}^{00} & \mathbf{1}^{00} \\
\hline \mathbf{1}^{0+} & \mathbf{1}^{00} & \mathbf{1}^{00}
\end{array}\right),
\end{aligned}
$$

where we have indicated U(1) charges with a subscript. This leads to the textures

$$
\begin{aligned}
M_{L R} & =\frac{v}{\sqrt{2}}\left(\begin{array}{ccc}
b_{1} \epsilon & 0 & 0 \\
0 & b_{2} \epsilon & b_{3} \epsilon \\
b_{4} \rho \epsilon^{\prime} & b_{5} \rho & b_{6} \rho
\end{array}\right), \quad \text { and } \\
M_{R R} & =\left(\begin{array}{ccc}
c_{1} \epsilon^{\prime} M_{F} & c_{2} \epsilon^{\prime} M_{F} & c_{3} \epsilon^{\prime} M_{F} \\
c_{2} \epsilon^{\prime} M_{F} & M_{22} & M_{23} \\
c_{3} \epsilon^{\prime} M_{F} & M_{23} & M_{33}
\end{array}\right) .
\end{aligned}
$$

Here the $b_{i}$ and $c_{i}$ are $\mathcal{O}(1)$ coefficients. Since the elements labeled $M_{22}, M_{23}$ and $M_{33}$ in $M_{R R}$ are each flavor-group invariant, they do not necessarily have to be at the same scale as $M_{F}$, or as each other. For the purposes of demonstrating the viability of the neutrino sector, we will take these elements to be at the scale $\epsilon^{\prime} M_{F}$, so that $M_{R R}$ takes the form

$$
M_{R R}=\epsilon^{\prime} M_{F}\left(\begin{array}{ccc}
c_{1} & c_{2} & c_{3} \\
c_{2} & c_{4} & c_{5} \\
c_{3} & c_{5} & c_{6}
\end{array}\right) \equiv \epsilon^{\prime} M_{F} \tilde{M}_{R R}
$$

In other words, with this choice, the right-handed Majorana matrix is a complete arbitrary matrix with $\mathcal{O}(1)$ entries, $\tilde{M}_{R R}$, times the scale $\epsilon^{\prime} M_{F}$. The Dirac mass matrix also has considerable freedom. Noting that our earlier fits indicated $\rho \approx \mathcal{O}(\epsilon)$, we can redefine the coefficients $b_{5}$ and $b_{6}$, and drop the 13 entry, which is higher order. Then we see that $M_{L R}$ is approximately of the form 
$M_{L R} \approx \frac{v \epsilon}{\sqrt{2}}\left(\begin{array}{ccc}b_{1} & 0 & 0 \\ 0 & b_{2} & b_{3} \\ 0 & b_{5} & b_{6}\end{array}\right) \equiv \frac{v \epsilon}{\sqrt{2}}\left(\begin{array}{cc}b_{1} & 0 \\ 0 & \tilde{Y}_{L R}\end{array}\right)$,

where $\tilde{Y}_{L R}$ is an arbitrary, two-by-two matrix with $\mathcal{O}(1)$ entries. The 10 free parameters in Eqs. (4.5) and the approximation shown in (4.6) are more than sufficient to obtain the desired values of $\Delta m_{32}^{2} / \Delta m_{21}^{2}$, as well as $\sin ^{2} \theta_{12}, \sin ^{2} \theta_{13}$ and $\sin ^{2} \theta_{23}$, while maintaining $\mathcal{O}(1)$ operator coefficients. The dependence of the output on products of the coefficients allows numerical values like 33 (the experimental value of $\Delta m_{32}^{2} / \Delta m_{21}^{2}$ ) or $1 / 33$ (very close to $\theta_{13}^{2}$ ) to arise without fine tunings. We note that the form of Eq. (4.6), with a nonvanishing 1-1 entry, is a consequence of the different charge assignment for the first-generation right-handed neutrino field. This entry of $M_{L R}$ originates from a charge conjugated $\mathbf{2}^{0+}$ flavon; in $T^{\prime}$, as in $\mathrm{SU}(2), \mathbf{2} \sim i \sigma^{2} \mathbf{2}^{*}$, which flips the relative location of the doublet vev in the first two columns of $M_{L R}$.

An example of a viable parameter set for the neutrino sector is shown in Table V. The neutrino mixing angles are defined via a standard parametrization of the PontecorvoMaki-Nakagawa-Sakata (PMNS) matrix, which we call $U$ below,

$$
U=U_{e}^{\dagger} U_{\nu}
$$

where $U_{e}\left(U_{\nu}\right)$ is a unitary matrix that diagonalizes the charged lepton (left-handed Majorana) matrix following our earlier convention, i.e., $M_{L L}=U_{\nu} M_{L L}^{\text {diag }} U_{\nu}^{\dagger}$. We can extract the mixing angles via the relations

$$
\begin{aligned}
& \sin ^{2} \theta_{13}=U_{13}^{2}, \quad \sin ^{2} \theta_{23}=U_{23}^{2} /\left(1-U_{13}^{2}\right) \quad \text { and } \\
& \sin ^{2} \theta_{12}=U_{12}^{2} /\left(1-U_{13}^{2}\right) .
\end{aligned}
$$

For the purpose of illustration, we fix $\epsilon, \epsilon^{\prime}$ and $\rho$, as well as the coefficients $l_{i}$ appearing in the charged lepton Yukawa

TABLE V. Example of a viable parameter choice for the neutrino sector.

\begin{tabular}{lcc}
\hline \hline \multicolumn{3}{c}{ Parameters } \\
\hline \multicolumn{2}{c}{$\epsilon=2.42 \times 10^{-2}, \epsilon^{\prime}=9.75 \times 10^{-5}, \rho=-1.38 \times 10^{-2}$} \\
\hline$b_{1}=1.66$ & $b_{2}=1.07$ & $b_{3}=2.10$ \\
$b_{4}=1.11$ & $b_{5}=-0.891$ & $b_{6}=1.61$ \\
$c_{1}=2.91$ & $c_{2}=1.04$ & $c_{3}=0.662$ \\
$c_{4}=1.21$ & $c_{5}=1.37$ & $c_{6}=1.44$ \\
Observable & Expt. value from [21] & Fit value \\
$\Delta m_{32}^{2}$ & $33.3 \pm 1.03$ & 33.8 \\
$\Delta m_{21}^{2}$ & $0.307 \pm 0.013$ & 0.307 \\
$\sin ^{2} \theta_{12}$ & $0.417 \pm 0.025$ & 0.444 \\
$\sin ^{2} \theta_{23}$ & $(2.12 \pm 0.08) \times 10^{-2}$ & $2.11 \times 10^{-2}$ \\
$\sin ^{2} \theta_{13}$ &
\end{tabular}

matrix, to the values that were obtained in our previous global fit of the charged fermions, Table II. A viable choice of neutrino sector parameters $b_{i}$ and $c_{i}$ is presented in Table V. These were obtained by defining a $\tilde{\chi}_{\nu}^{2}$ for the neutrino sector that takes into account the neutrino observables listed in the table and also places weight on the neutrino-sector coefficients being $\mathcal{O}(1)$, in analogy to our approach in the charged fermions. This function can be used to diagnose when a good-enough parameter choice has been obtained.

Since the right-handed neutrino mass scale is set by $\epsilon^{\prime} M_{F}$, the neutrino mass squared differences (rather than the ratio) can be used to determine the flavor scale. Using either experimental value [21]

$$
\begin{aligned}
& \Delta m_{21}^{2}=(7.53 \pm 0.18) \times 10^{-5} \mathrm{eV}^{2} \quad \text { or } \\
& \triangle m_{32}^{2}=(2.51 \pm 0.05) \times 10^{-3} \mathrm{eV}^{2},
\end{aligned}
$$

we find that the solution in Table $\mathrm{V}$ corresponds to

$$
M_{F}=4.6 \times 10^{16} \mathrm{GeV} .
$$

This is consistent with our axiflavon constraint in Eq. (3.20).

Finally, it is worth pointing out that the predictions of flavor models with small symmetry-breaking parameters in the effective field theory approach come in the form of powers of these small parameters. What is remarkable about the present construction is that we have arranged the final left-handed neutrino mass matrix to be immune from the effects of these small parameters which give us the hierarchies of the charged fermions, even though many of the neutrino fields are nontrivially charged under the flavor group. All that is left are order-one operator coefficients, which may simply be random (in the spirit of neutrino anarchy [35]) or fixed by other physics in the ultraviolet.

\section{CONCLUSIONS}

In this paper, we have studied a nonsupersymmetric flavor model based on the double tetrahedral group, $T^{\prime}$. Improving on earlier work by Carone, Chaurasia and Vasquez [11], we formulate a simpler model that dispenses with the triplet flavon $S$ and eliminates some small numerical coefficients that were assumed in one version of the model to arise from unspecified physics at higher energy scales. Moreover, by replacing one of the Abelian discrete group factors by a continuous U(1) flavor symmetry, we endow the theory with a flavorful axion that solves the strong $C P$ problem. The flavorful axion decay constant $f_{a}$ is related to the flavor scale $M_{F}$ (the cut off of the effective theory) and falls roughly two orders of magnitude beneath it. We present constraints on $f_{a}$ coming from FCNC processes and find that the strongest lower bound comes from the process $K^{+} \rightarrow \pi^{+} a$, yielding 
$f_{a}>1.2 \times 10^{11} \mathrm{GeV}$. We show that the Yukawa matrices predicted by the model provide a good fit to the observed charged fermion masses and mixing angles, taking into account the running from the flavor scale down to the weak scale. We then successfully extend the model to the neutrino sector, by introducing three generations of right-handed neutrinos and employing a Type-I seesaw mechanism to explain the smallness of the light neutrino masses. By charging only the first generation right-handed neutrino nontrivially under $T^{\prime}$, we show how the mass matrix for the light neutrino mass eigenstates, which must account for two large mixing angles and requires only a modest hierarchy between the neutrino masses, can be predicted by the same theory that yields the strong hierarchies of the charged fermion Yukawa matrices. For the particular extension to the neutrino sector presented here, the flavor scale is roughly five orders of magnitude higher than what is required to satisfy the flavorful axion bounds. This suggests that flavor-changing signals from the flavorful axion will not be easily observable unless additional symmetries are introduced to lower the scale associated with the right-handed neutrinos.

\section{ACKNOWLEDGMENTS}

We thank Tangereen Claringbold for useful discussions and Shikha Chaurasia for cross checking some of our numerical results. We thank the NSF for support under Grant No. PHY-1819575.
[1] A. Aranda, C. D. Carone, and R. F. Lebed, U(2) flavor physics without U(2) symmetry, Phys. Lett. B 474, 170 (2000).

[2] A. Aranda, C. D. Carone, and R. F. Lebed, Maximal neutrino mixing from a minimal flavor symmetry, Phys. Rev. D 62, 016009 (2000).

[3] R. Barbieri, G. R. Dvali, and L. J. Hall, Predictions from a U(2) flavor symmetry in supersymmetric theories, Phys. Lett. B 377, 76 (1996).

[4] R. Barbieri, L. J. Hall, S. Raby, and A. Romanino, Unified theories with U(2) flavor symmetry, Nucl. Phys. B493, 3 (1997).

[5] P. H. Frampton and T. W. Kephart, Simple nonAbelian finite flavor groups and fermion masses, Int. J. Mod. Phys. A 10, 4689 (1995).

[6] I. Girardi, A. Meroni, S. T. Petcov, and M. Spinrath, Generalised geometrical $C P$ violation in a $T^{\prime}$ lepton flavour model, J. High Energy Phys. 02 (2014) 050; A. Meroni, S. T. Petcov, and M. Spinrath, A SUSY SU $(5) \times \mathrm{T}^{\prime}$ unified model of flavour with large $\theta_{13}$, Phys. Rev. D 86, 113003 (2012); M. C. Chen, J. Huang, K. T. Mahanthappa, and A. M. Wijangco, Large $\theta_{13}$ in a $\operatorname{SUSY} \operatorname{SU}(5) \times \mathrm{T}^{\prime}$ model, J. High Energy Phys. 10 (2013) 112; P. H. Frampton, C. M. Ho, and T. W. Kephart, Heterotic discrete flavor model, Phys. Rev. D 89, 027701 (2014); Y. H. Ahn, Leptons and quarks from a discrete flavor symmetry, Phys. Rev. D 87, 113011 (2013); A. Meroni, E. Molinaro, and S. T. Petcov, Revisiting Leptogenesis in a SUSY SU $(5) \times \mathrm{T}^{\prime}$ model of flavour, Phys. Lett. B 710, 435 (2012); D. A. Eby and P. H. Frampton, Nonzero $\theta_{13}$ signals nonmaximal atmospheric neutrino mixing, Phys. Rev. D 86, 117304 (2012); Dark matter from binary tetrahedral flavor symmetry, Phys. Lett. B 713, 249 (2012); D. A. Eby, P. H. Frampton, X. G. He, and T. W. Kephart, Quartification with $T^{\prime}$ Flavor, Phys. Rev. D 84, 037302 (2011); M. C. Chen, K. T. Mahanthappa, and F. Yu, A viable Randall-Sundrum model for quarks and leptons with $T^{\prime}$ family symmetry,
Phys. Rev. D 81, 036004 (2010); M. C. Chen and K. T. Mahanthappa, Group theoretical origin of $C P$ violation, Phys. Lett. B 681, 444 (2009); P. H. Frampton, T. W. Kephart, and S. Matsuzaki, Simplified renormalizable $T^{\prime}$ model for tribimaximal mixing and Cabibbo angle, Phys. Rev. D 78, 073004 (2008); C. Luhn, Discrete anomalies of binary groups, Phys. Lett. B 670, 390 (2009); G. J. Ding, Fermion mass hierarchies and flavor mixing from $T^{\prime}$ symmetry, Phys. Rev. D 78, 036011 (2008); S. Sen, Quark masses in supersymmetric $\mathrm{SU}(3)_{C} \times \mathrm{SU}(3)_{W} \times \mathrm{U}(1)_{X}$ model with discrete $T^{\prime}$ flavor symmetry, Phys. Rev. D 76, 115020 (2007); A. Aranda, Neutrino mixing from the double tetrahedral group $T^{\prime}$, Phys. Rev. D 76, 111301 (2007); P. H. Frampton and T. W. Kephart, Flavor symmetry for quarks and leptons, J. High Energy Phys. 09 (2007) 110; M. C. Chen and K. T. Mahanthappa, CKM and tribimaximal MNS matrices in a $S U(5) \times{ }^{(d)} T$ model, Phys. Lett. B 652, 34 (2007); F. Feruglio, C. Hagedorn, Y. Lin, and L. Merlo, Tri-bimaximal neutrino mixing and quark masses from a discrete flavour symmetry, Nucl. Phys. B775, 120 (2007); B836, 127(E) (2010).

[7] S. Abel, K. R. Dienes, and E. Mavroudi, Towards a nonsupersymmetric string phenomenology, Phys. Rev. D 91, 126014 (2015).

[8] P. W. Graham, D. E. Kaplan, and S. Rajendran, Cosmological Relaxation of the Electroweak Scale, Phys. Rev. Lett. 115, 221801 (2015).

[9] N. Arkani-Hamed, T. Cohen, R. T. D’Agnolo, A. Hook, H. D. Kim, and D. Pinner, Solving the Hierarchy Problem at Reheating with a Large Number of Degrees of Freedom, Phys. Rev. Lett. 117, 251801 (2016).

[10] For example, S. Weinberg, Anthropic Bound on the Cosmological Constant, Phys. Rev. Lett. 59, 2607 (1987).

[11] C. D. Carone, S. Chaurasia, and S. Vasquez, Flavor from the double tetrahedral group without supersymmetry, Phys. Rev. D 95, 015025 (2017). 
[12] F. Bjrkeroth, E. J. Chun, and S. F. King, Flavourful axion phenomenology, J. High Energy Phys. 08 (2018) 117.

[13] Y. Ema, K. Hamaguchi, T. Moroi, and K. Nakayama, Flaxion: A minimal extension to solve puzzles in the standard model, J. High Energy Phys. 01 (2017) 096.

[14] L. Calibbi, F. Goertz, D. Redigolo, R. Ziegler, and J. Zupan, Minimal axion model from flavor, Phys. Rev. D 95, 095009 (2017).

[15] M. Linster and R. Ziegler, A Realistic $U(2)$ model of flavor, J. High Energy Phys. 08 (2018) 058.

[16] F. Arias-Aragon and L. Merlo, The minimal flavour violating axion, J. High Energy Phys. 10 (2017) 168.

[17] F. Wilczek, Axions and Family Symmetry Breaking, Phys. Rev. Lett. 49, 1549 (1982).

[18] Z. G. Berezhiani and M. Y. Khlopov, Cosmology of spontaneously broken gauge family symmetry, Z. Phys. C 49, 73 (1991); C. Q. Geng and J. N. Ng, Flavor connections and neutrino mass hierarchy invariant invisible axion models without domain wall problem, Phys. Rev. D 39, 1449 (1989); K. S. Babu and S. M. Barr, Family symmetry, gravity, and the strong $C P$ problem, Phys. Lett. B 300, 367 (1993); C. S. Fong and E. Nardi, Spontaneous Breaking of Flavor Symmetry Avoids the Strong CP Problem, Phys. Rev. Lett. 111, 061601 (2013); M. E. Albrecht, T. Feldmann, and T. Mannel, Goldstone bosons in effective theories with spontaneously broken flavour symmetry, J. High Energy Phys. 10 (2010) 089; Y. H. Ahn, Flavored Peccei-Quinn symmetry, Phys. Rev. D 91, 056005 (2015).

[19] Y. H. Ahn, Compact model for quarks and leptons via flavored-axions, Phys. Rev. D 98, 035047 (2018).

[20] A. Falkowski, M. Nardecchia, and R. Ziegler, Lepton flavor non-universality in B-meson decays from a U(2) flavor model, J. High Energy Phys. 11 (2015) 173.

[21] M. Tanabashi et al. (Particle Data Group), Review of particle physics, Phys. Rev. D 98, 030001 (2018).

[22] L. M. Krauss and F. Wilczek, Discrete Gauge Symmetry in Continuum Theories, Phys. Rev. Lett. 62, 1221 (1989).
[23] J. E. Kim, Light pseudoscalars, particle physics and cosmology, Phys. Rep. 150, 1 (1987).

[24] D. J. E. Marsh, Axion cosmology, Phys. Rep. 643, 1 (2016).

[25] J. L. Feng, T. Moroi, H. Murayama, and E. Schnapka, Third generation familons, b factories, and neutrino cosmology, Phys. Rev. D 57, 5875 (1998).

[26] M. Bauer, T. Schell, and T. Plehn, Hunting the flavon, Phys. Rev. D 94, 056003 (2016).

[27] A. Celis, J. Fuentes-Martin, and H. Serodio, An invisible axion model with controlled FCNCs at tree level, Phys. Lett. B 741, 117 (2015).

[28] M. E. Albrecht, T. Feldmann, and T. Mannel, Goldstone bosons in effective theories with spontaneously broken flavour symmetry, J. High Energy Phys. 10 (2010) 089.

[29] A. Davidson and M. A.H. Vozmediano, The horizontal axion alternative: The interplay of vacuum structure and flavor interactions, Nucl. Phys. B248, 647 (1984).

[30] S. Adler et al. (E949 and E787 Collaborations), Measurement of the $K^{+} \rightarrow \pi^{+} \nu \nu$ branching ratio, Phys. Rev. D 77, 052003 (2008).

[31] J. K. Ahn et al. (KOTO Collaboration), A new search for the $K_{L} \rightarrow \pi^{0} \nu \bar{\nu}$ and $K_{L} \rightarrow \pi^{0} X^{0}$ decays, Prog. Theor. Exp. Phys. 2017, $021 \mathrm{C} 01$ (2017).

[32] R. Ammar et al. (CLEO Collaboration), Search for the Familon via $B^{+-} \rightarrow \pi^{ \pm} X^{0}, B^{ \pm} \rightarrow K^{ \pm} X^{0}$, and $B^{0} \rightarrow K_{S}^{0} X^{0}$ Decays, Phys. Rev. Lett. 87, 271801 (2001).

[33] R. Bayes et al. (TWIST Collaboration), Search for two body muon decay signals, Phys. Rev. D 91, 052020 (2015).

[34] H. Albrecht et al. (ARGUS Collaboration), A search for lepton flavor violating decays $\tau \rightarrow e \alpha, \tau \rightarrow \mu \alpha$, Z. Phys. C 68, 25 (1995).

[35] L. J. Hall, H. Murayama, and N. Weiner, Neutrino Mass Anarchy, Phys. Rev. Lett. 84, 2572 (2000). 